\section{Hyperfine Structure of the Resonance Lines of Silver}

THE structure of the resonance lines of silver has been investigated by the method of absorption in an atomic beam, the high resolving power instrument being a Fabry Perot étalon with plate separations between $2 \mathrm{~cm}$. and $10 \mathrm{~cm}$. Each line was found to possess four components ; for the line $5 S_{1 / 2}-5{ }^{2} P_{3 / 2}$, $3281 \mathrm{~A}$., the positions were $0.000,-0.013,-0.052$ and $-0.077 \mathrm{~cm}^{-1}$, and for $5 S_{1 / 2}-5{ }^{2} P_{1 / 2}, 3383 \mathrm{~A}$., they were $0.000,-0.013,-0.058$ and $-0.084 \mathrm{~cm}^{-1}$. In both lines the two components of shorter wave. length were nearly equal in intensity and very much stronger than the two of longer wave-length, which were also of nearly equal intensity; photometer curves of the absorption showed that the intensity ratio of the strong lines to the weak lines was approximately $3: 1$.

As the structure is nearly the same in both resonance lines, it must be due mainly to the common level $5 S_{1 / 2}$. Silver consists of two isotopes, 107 and 109 , their abundancy ratio being about $1 \cdot 3: 1$. Each isotope must therefore give rise to one of the strong components and one of the weak components, the intensity ratio being $3: 1$; from this it follows that the nuclear spin of both isotopes is $\frac{1}{2}$.

The difference in abundancy of the two isotopes is small, and the two strong lines (and also the two weak lines) are nearly equal in intensity; however, the photometer curves showed that the component at $-0.013 \mathrm{~cm}^{-1}$ was rather stronger than that at $0.000 \mathrm{~cm} .^{-1}$ and that at $-0.052 \mathrm{~cm} .^{-1}$ was stronger than that at $-0.077 \mathrm{~cm}^{-1}$. It therefore appears probable that $-0.013 \mathrm{~cm} .^{-1}$ and $-0.052 \mathrm{~cm} .^{-1}$ are due to ${ }^{107} \mathrm{Ag}$ and $0.000 \mathrm{~cm} .^{-1}$ and $-0.077 \mathrm{~cm}^{-1}$ are due to ${ }^{109} \mathrm{Ag}$. On this assumption the nuclear magnetic moments, calculated from Goudsmid's formula ${ }^{1}$ for the splitting of an $S_{1 / 2}$ term, are -0.10 nuclear magneton for ${ }^{102} \mathrm{Ag}$ and -0.19 nuclear magneton for ${ }^{109} \mathrm{Ag}$. There is also a small isotope shift, the centre of gravity of the lines of ${ }^{107} \mathrm{Ag}$ being displaced by about $+0.004 \mathrm{~cm} .^{-1}$.

The difficulty of measuring the small difference in intensity of the very close lines 0.000 and $0.013 \mathrm{~cm}^{-1}$ is very great, so that the possibility that 0.000 and $-0.052 \mathrm{~cm}^{-1}$ belong to one isotope and -0.013 and $-0.077 \mathrm{~cm} .^{-1}$ to the other, is not quite excluded ; in this case the nuclear spins would still be $\frac{1}{2}$, but the magnetic moments would be -0.13 and -0.16 nuclear magneton, and the isotope shift $0 \cdot 014$ cm..$^{-1}$.

A doublet structure observed by $\mathrm{Hill}^{2}$ is in agreement with the above result, the small separations being unresolved on account of the very much greater Doppler width of the lines given by the hollow cathode tube which he used; in order to resolve the smallest separation, the temperature of the tube would have needed to be about $15^{\circ} \mathrm{Abs}$. The intensity ratio which he observed was falsified by incomplete resolution, the Doppler wing of the strong component overlapping the weak component; if this is allowed for, the intensity ratio is in agreement with the value $3: 1$.

Clarendon Laboratory,

D. A. JACKSON

H. KUHN.

Oxford.

May 8.

1. Goudsmid, Phys. Rev., 43, 636 (1933)

1H. Hill, Phys. Rev., 48, 233 (1935).
Anomalies in the Fine Structure of the First Spark Spectrum of Iodine

THRee years ago, the multiplet and hyperfine structures of the ${ }^{4} S$-system of the first spark spectrum of iodine were analysed by $\mathrm{me}^{1}$, and I deduced the nuclear spun of iodine to be 5/2. Recently, Lacroute ${ }^{2}$ has published an extensive multiplet classification of the ${ }^{2} D$-system, and established numerous terms. It has now become possible to construct the hyperfine structure scheme of this system. I wish to report here remarkable anomalies in the fine structure of certain terms of the ${ }^{2} D$-system.

The structure of the line $\lambda 5678 \cdot 1$ classified by Lacroute as $\left({ }^{2} D\right) 6 s^{3} D_{2}-\left({ }^{2} D\right) 6 p^{3} F_{2}$ is given in Fig. 1 . The fine structure intervals in the term $\left({ }^{2} D\right) 68^{3} D_{2}$ are
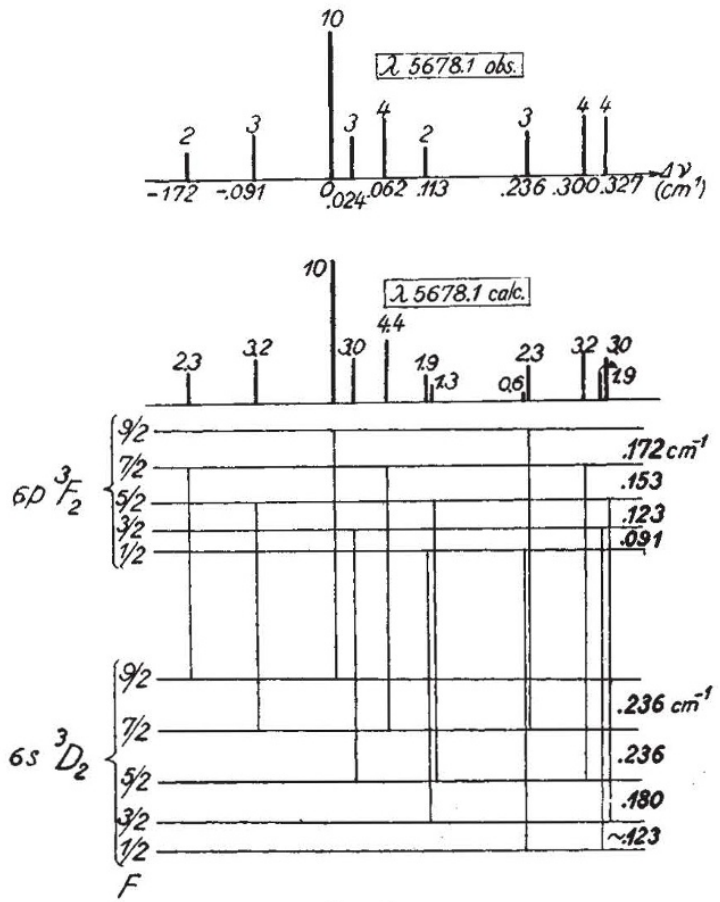

Fia. 1.

irregular and do not obey Landé's interval rule. Next, the line $\lambda 4060 \cdot 2$ classified by me as $\left({ }^{4} S\right) 5 d^{3} D_{0}$ $\left({ }^{2} D\right) 6 p^{3} D_{1}$ consists of three components :

$$
0.000(4), \quad+0.081(3), \quad+0.121(2) \mathrm{cm}^{-1} \text {. }
$$

Numbers in parentheses represent the intensities. Since the $j$-value of the lower term is 0 , the intervals of the components give directly the fine structure intervals of the upper term $\left({ }^{2} D\right) 6 p^{3} D_{1}$. Here the $F 5 / 2-7 / 2$ interval is smaller than the $F 3 / 2-5 / 2$ interval, a phenomenon which has never been ob. served in any term of another spectrum.

One might, at first sight, interpret such a breakdown of Landé's interval rule as due to perturbation by terms lying very near to $\left({ }^{2} D\right) 6 s^{3} D_{2}$ or $\left({ }^{2} D\right) 6 p^{3} D_{2}$. But I have failed to discover such terms. On the other hand, Casimir' has proposed the theory of nuclear electric moment, in order to account for a small systematic deviation from Landé's interval rule. Further extension of his theory will perhaps be able to account, at least qualitatively, for the re. markable anomalies in the iodine spark spectrum.

It may be noted that the data given here are not in agreement with those recently published by 